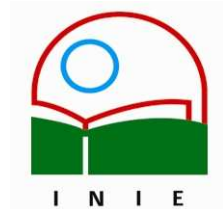

Actualidades Investigativas en Educación

Revista Electrónica publicada por el

Instituto de Investigación en Educación

Universidad de Costa Rica

ISSN 1409-4703

http://revista.inie.ucr.ac.cr

COSTA RICA

\title{
ACERCAMIENTOS TEÓRICOS PARA DEFINIR LA CALIDAD DE LA ENSEÑANZA DE LA LENGUA ESPAÑOLA EN LA EDUCACIÓN PRIMARIA COSTARRICENSE
}

THEORETICAL APPROACHES TO DEFINE THE QUALITY OF TEACHING OF THE SPANISH LANGUAGE IN THE COSTA RICAN PRIMARY EDUCATION

\author{
Volumen 8, Número 1 \\ pp. 1-22
}

Este número se publicó el 30 de abril 2008

Marielos Murillo Rojas

La revista está indexada en los directorios:

LATINDEX, REDALYC, IRESIE, CLASE, DIALNET, DOAJ, E-REVIST@S,

La revista está incluida en los sitios:

REDIE, RINACE, OEI, MAESTROTECA, HUASCARAN

Los contenidos de este artículo están bajo una licencia Creative Commons

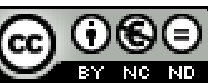




\title{
ACERCAMIENTOS TEÓRICOS PARA DEFINIR LA CALIDAD DE LA ENSEÑANZA DE LA LENGUA ESPAÑOLA EN LA EDUCACIÓN PRIMARIA COSTARRICENSE
}

\author{
THEORETICAL APPROACHES TO DEFINE THE QUALITY OF TEACHING OF THE
} SPANISH LANGUAGE IN THE COSTA RICAN PRIMARY EDUCATION

Marielos Murillo Rojas ${ }^{1}$

\begin{abstract}
Resumen: Este artículo aporta material de discusión sobre los aspectos por considerar al definir la calidad de la enseñanza de la lengua materna en la educación primaria. Por tanto, desarrolla los siguientes aspectos: formación docente en el área de lengua, programación del aprendizaje lingüístico escolar, enriquecimiento de la competencia comunicativa, políticas de supervisión y acompañamiento al ejercicio profesional docente y recursos didácticos graduados, pertinentes y accesibles. También define algunas tareas pendientes a cargo del Ministerio de Educación Pública y las universidades estatales.
\end{abstract}

Palabras claves: calidad de la educación, enseñanza de la lengua española, educación primaria.

Abstract: This article contributes with some discussion material on the aspects to consider when defining the quality of the teaching of the maternal language in primary education. Therefore, it develops the following aspects: educational formation in the language area, programming of the scholastic linguistic learning, enrichment of the communicative competition, and supervision and support policies to the educational professional practice and pertinent, accessible and graduated didactic resources. Also, it defines some pending tasks in charge of the Ministry of Public Education and the state universities.

Key words: quality of the education, teaching of the Spanish language, primary education.

\section{Introducción}

El sexto objetivo en materia de Educación para Todos, Dakar, 2000, persigue "mejorar todos los aspectos cualitativos de la educación, garantizando los parámetros más elevados, para conseguir resultados de aprendizaje reconocidos y mensurables, especialmente en lectura, escritura, aritmética y competencias prácticas esenciales para la vida diaria" (UNESCO, 2005, p. 30).

Este objetivo evidencia la necesidad de que los ciudadanos cuenten con una formación de base e instrumental, que les permita desarrollar capacidades cognoscitivas superiores y competencias psicosociales para desenvolverse adecuadamente en diferentes contextos.

\footnotetext{
${ }^{1}$ Doctora en Formación de Profesorado área Didáctica de la Lengua Española, de la Universidad de Extremadura, España. Máster en Lingüística; Licenciada en Educación Preescolar, ambos títulos de la Universidad de Costa Rica. Profesora de la Escuela de Formación Docente, Universidad de Costa Rica.
}

Dirección electrónica: marielosmuro@yahoo.es

Artículo recibido: 9 de noviembre, 2007

Aprobado: 31 de marzo, 2008 
En consecuencia, la enseñanza de la lengua materna como área instrumental para la socialización y adquisición de nuevos conocimientos, adquiere niveles de importancia que sobrepasan la dedicación de dos lecciones diarias, en el caso de la educación primaria, y se constituye en un eje pedagógico, pues posibilita todo tipo de aprendizaje formal e informal.

Ahora bien, y con el propósito de lograr ese objetivo, las macrohabilidades lingüísticas expresión oral, comprensión oral, lectura y escritura- deben emerger en un ambiente que tenga como meta enriquecer la competencia comunicativa del educando, a fin de dotarlo de competencias básicas para la vida.

La necesidad de desarrollar en el alumnado competencias esenciales para la vida, junto con el dominio creciente de la lectura y la escritura como conocimientos instrumentales que aseguren el desenvolvimiento óptimo en los ámbitos académicos, sociales y culturales, conlleva necesariamente el replanteamiento de los aspectos que definirán la calidad de la enseñanza de la lengua en la educación primaria costarricense. Por tanto, este artículo tiene como objetivo definir el concepto de calidad educativa en el contexto de la educación primaria costarricense y proponer algunos criterios que podrían considerarse para definir la calidad en ese nivel educativo.

\section{Concepto de calidad educativa}

En el Informe de Seguimiento de Educación para Todos en el Mundo. El imperativo de la Calidad, UNESCO, 2005, se señala que "dos principios caracterizan la mayoría de las tentativas de definición de lo que es una educación de calidad: el primero considera que el desarrollo cognitivo del educando es el objetivo explícito más importante de todo sistema educativo y, por consiguiente, su éxito en este ámbito constituye un indicador de su calidad; el segundo hace hincapié en el papel que desempeña la educación en la promoción de las actitudes y los valores relacionados con una buena conducta cívica, así como en la creación de condiciones propicias para el desarrollo afectivo y creativo del educando. (p. 18)

Ahora bien, con el propósito de conciliar los diferentes enfoques existentes sobre calidad educativa, se adoptan como criterios de calidad cinco aspectos, a saber: "los educandos, cuya diversidad es preciso tener en cuenta; el contexto socioeconómico nacional; los recursos materiales y humanos; el proceso de enseñanza y aprendizaje; los resultados y beneficios de la educación" (p. 21). 
La interacción de estos aspectos permitirá entender qué es la calidad educativa y, en consecuencia, dar seguimiento a las tareas conducentes al logro de una educación contextualizada, pertinente y de calidad, según los parámetros propuestos en las diferentes realidades educativas.

Por otra parte, definir la calidad de la educación implica considerar cuál es la corriente teórica que sustenta las políticas educativas, operacionalizada en objetivos o metas, en las diferentes realidades educativas; por tanto, desde el humanismo, el conductismo, la pedagogía crítica, los enfoques autóctonos y los enfoques de educación de adultos, se conceptualizará la calidad educativa.

En Costa Rica, por ejemplo, la Política Educativa vigente señala explícitamente como fuentes filosóficas el humanismo, el racionalismo y el constructivismo, los cuales aportarán, desde sus especificidades, elementos básicos para promover el desarrollo integral del educando. Sin embargo, no se definen explícitamente los criterios para valorar la calidad de la educación que se ofrece, por lo que con este marco de referencia es difícil orientar cualitativamente la educación nacional y la correspondiente valoración de su calidad.

Indudablemente, los educandos y los docentes se constituyen en elementos claves de toda política educativa tendiente a alcanzar parámetros de calidad. El educando tiene derecho a aprender en condiciones adecuadas y bajo un marco pedagógico que privilegia su desarrollo integral. El docente debe poseer una formación y condiciones personales tales que le permitan conducir adecuadamente los procesos educativos de los escolares en pro de su desarrollo integral.

En vista de la relevancia del papel del educador, se aboga como demanda general por una mejor formación inicial y continua, a lo largo del ejercicio profesional docente. "Los países que han conseguido alcanzar normas elevadas en materia de aprendizaje son los que han invertido constantemente en la mejora de la profesión docente" (p.19).

Otro de los aspectos por considerar es la definición de las áreas básicas objeto de estudio. En este sentido cabría preguntarse qué competencias debe poseer una persona para transitar con éxito en la era de la información y el cambio constante. Al respecto Adalberto Ferrández (citado por Marcelo, 2004) 
insistió a lo largo de su vida en la importancia de entender la formación de base como el sustrato imprescindible para cualquier propuesta formativa con opciones de éxito. (... ) La formación de base debe aspirar a permanecer en un mundo donde todo cambia, a dotar a las personas de autonomía personal, capacidad de comunicación, conocimiento de los procesos de resolución de problemas, manejo de la información, etc. (p. 541)

En este sentido, en el informe supracitado se considera el aprendizaje de la lectura y la escritura como medios esenciales para construir otros aprendizajes curriculares y como instrumentos fundamentales para formular previsiones sobre los resultados del aprendizaje a largo plazo. "La lectura debe considerarse un área prioritaria a la hora de centrar los esfuerzos en la mejora de la calidad de la educación básica” (p.19).

Por otra parte, investigaciones que se han ocupado de los procesos de aprendizaje -esto es, en la interacción creativa de alumnos y docentes en el aula misma-

demuestran que las escuelas primarias de calidad se suelen caracterizar por un sólido espíritu de liderazgo; un entorno de la escuela y las aulas donde reinan el orden y la seguridad; un esfuerzo centrado en la adquisición de las competencias básicas; unas expectativas ambiciosas por lo que respecta a los resultados de los alumnos; y una evaluación frecuente de los progresos de éstos. También hay otros elementos que contribuyen a la calidad de la escuela: el grado de dominio del plan de estudios por parte de los docentes, su nivel de aptitud verbal y las esperanzas que tienen depositadas en sus alumnos. (p. 22)

Ahora bien, lograr una educación de calidad es la meta del nuevo milenio, reto que debe afrontar cada sociedad a partir de la definición del tipo de ciudadano que desea formar. En Costa Rica, creemos, se debe empezar por una revisión sustancial de la política educativa, marco jurídico que define la orientación de la planificación educativa a nivel macro y micro, y a partir de esta señalar explícitamente competencias básicas que serán objeto de trabajo en las aulas costarricenses.

Afortunadamente se cuenta hoy con resultados de investigaciones, realizadas por las universidades estatales en institutos de investigación -INIE, CIDE, INIL-y trabajos finales de graduación -licenciatura, maestría y doctorado- , que señalan, desde diferentes marcos 
teóricos, la situación actual de la enseñanza de la lengua española en la educación primaria; todos estos aportes deben considerarse antes de iniciar cualquier empresa de planificación curricular, con miras al logro de la calidad educativa (Murillo y Morales, 2007).

También podrían aprovecharse las experiencias aprendidas de los países que se destacan con niveles elevados en la calidad de la educación, tal es el caso de Canadá, Cuba, Finlandia y la República de Corea.

Estos países presentan tres características comunes. La primera atañe a los docentes. En todos esos países, se ha comprobado que existe una gran estima por la profesión docente, una sólida formación inicial y condiciones de admisión relativamente estrictas, un sistema bien desarrollado de formación permanente y una serie de mecanismos de aprendizaje mutuo y apoyo a los profesores. Aun cuando exista una escasez de docentes, no se hacen concesiones en cuanto a su calidad.

La segunda característica es la continuidad de las políticas. La República de Corea intentó deliberadamente neutralizar los efectos de los cambios políticos, estableciendo órganos consultivos. En Cuba, la continuidad es inherente al sistema político. Canadá y Finlandia cuentan con sólidas bases de conocimientos sobre la educación en el seno de las instituciones de formación y apoyo a los docentes, lo cual parece impedir a los gobiernos que cambien de orientación demasiado frecuente y radicalmente.

La tercera característica es el elevado nivel de compromiso público con la educación, que parece emanar de una sólida visión política. La determinación de la República de Corea a convertirse en un país competitivo a nivel mundial y mantener esa posición, la voluntad de Cuba de defender su revolución, la convicción del Canadá de que su fuerza como nación descansa en la diversidad cultural, y el profundo compromiso de Finlandia con el desarrollo humano y la igualdad, son elementos que, de distintas formas, han ejercido una influencia considerable en las políticas y los resultados educativos.

Otra característica común de la República de Corea y Cuba es el dinamismo excepcional de los alumnos, docentes y padres. En ambos países, va unido a una atmósfera de competitividad, aunque desde puntos de vista muy diferentes y de maneras muy distintas. 
"Saber si esto se puede reproducir en otros países en desarrollo, y de qué manera, es un interrogante que todavía no ha tenido respuesta" (pp. 58-59).

Respecto de la continuidad en las políticas educativas nacionales, emanadas desde el gobierno central, los costarricenses hemos sufrido el 'síndrome mi programa educativo es el mejor'; en consecuencia, cada gobierno desea imprimir su sello e introduce, regularmente, nuevos programas. Lo lamentable es que esos programas, generalmente, obvian la heterogeneidad que caracteriza a la escuela costarricense, no atienden las características evolutivas de los destinatarios potenciales -no hay una secuenciación adecuada-, algunos de los contenidos propuestos carecen de pertinencia y no se definen con claridad las competencias fundamentales por desarrollar en los escolares, por lo menos en el área de lengua.

Una muestra de lo señalado anteriormente se desarrollada ampliamente en el artículo "La enseñanza de la lengua española en la educación primaria costarricense. Período 19952007. Análisis crítico", (Murillo, 2007, p. 35), investigación que concluye, entre otras cosas:

Lamentablemente en los programas analizados -1995, 2001 y 2005- no se percibe ese aporte académico vario; al contrario pareciera que quienes se han ocupado de elaborar los programas de estudio del área de español carecen de una formación específica que les permita conjugar adecuadamente los aspectos señalados.

Respecto de cada programa específico se concluye lo siguiente:

El programa de 1995 muestra coherencia interna entre lo postulado como fundamento teórico y el desarrollo de las cuatro áreas del lenguaje -lectura, escritura, expresión oral y escucha- a lo largo de los programas específicos de cada año. Aspectos que permiten al educador, en su función de planificador del trabajo de aula, visualizar cuál es su responsabilidad como guía del proceso de enriquecimiento de la lengua materna de sus alumnos.

Pese a lo anterior, este programa se vio limitado por la incorporación de un enfoque de la lectura superado y de un tratamiento del aprestamiento que no corresponde al desarrollo de las prácticas pedagógicas vigentes desde ese momento. 
Es justo destacar el acierto en la elección del enfoque comunicativo como sustento del área de lengua y como corolario el tratamiento de los temas gramaticales en función de la producción textual (p. 19).

El programa de 2001 constituye un claro retroceso en lo avanzado a nivel de planificación lingüística curricular hasta la fecha, pues en vez de avanzar hacia una mejor aplicación del enfoque comunicativo en la enseñanza de la lengua, se retoma el tradicional método gramatical, el cual no responde a las particularidades del desarrollo del niño en la etapa escolar (p. 28).

El programa de 2005 no experimentó ningún reajuste en la propuesta de contenidos, como se indicó en la presentación del mismo, pero sí mejoró la redacción de los objetivos, en el sentido de que se tornaron más abarcadores, recuérdese que en el programa de 2001, segundo grado, se presentaron 97 y en el actual 26, manteniendo exacta la columna de contenidos.

Al terminar este apartado es necesario señalar que respecto del programa de 1995, la presente propuesta obvia el enfoque teórico que se ha venido manteniendo, explícito en las primeras páginas de los programas y que debería sustentar el trabajo propuesto para cada año escolar, y en su lugar da cabida al tradicional enfoque gramatical. (p. 33)

Como puede observarse, no ha habido continuidad en programas pertenecientes a una misma política educativa (Hacia el Siglo XXI), lo que atenta contra la calidad de la enseñanza, en este caso de la lengua materna.

Por otra parte, y refiriéndonos a la tercera característica que ostentan los programas de calidad: compromiso público con la educación, es imperativo, en el caso costarricense, revisar el compromiso asumido con la formación inicial docente, la cual no responde a políticas nacionales y carece de una rectoría que la regule (Sol, 2004).

En ese sentido, se recomienda, con carácter de emergencia, formar un ente rector con competencias para generar políticas de reacreditación de los profesionales que ejerzan la educación y denegar la continuidad en el ejercicio de aquellos que no califican para tal labor. 
En vista de lo anterior, y si se desea ofrecer una educación de calidad, Costa Rica debe empezar por resolver el problema de la formación inicial docente, considerando como mínimo: la diversidad de planes de formación que ofrecen las universidades (públicas y privadas), los perfiles profesionales de los formadores de formadores y los criterios de selección de los futuros docentes. Simultáneamente debe trabajarse con un plan de desarrollo educativo pertinente y que prometa claridad en cuanto a las competencias fundamentales que debe asegurar la educación obligatoria.

\section{Criterios por considerar en la calidad de la enseñanza de la lengua española en la educación primaria}

A continuación se señalan los aspectos que facilitarían un abordaje de calidad en el área de lengua española en la educación primaria costarricense:

1. Docentes con una fuerte formación en el área de lengua.

2. Programación del aprendizaje lingüístico escolar, en atención a las competencias por desarrollar en cada nivel educativo.

3. Práctica profesional docente que privilegie el enriquecimiento de la competencia comunicativa de los escolares, mediante la promoción de aprendizajes relevantes

5. Políticas claras de supervisión y acompañamiento del ejercicio profesional docente.

4. Recursos didácticos graduados, pertinentes y accesibles

Seguidamente, se desarrolla de forma sucinta cada uno de los aspectos mencionados.

\subsection{Docentes con una fuerte formación en el área de lengua}

En primera instancia nos referiremos sucintamente a la formación que ofrecen las universidades estatales en el área de lengua española.

Los programas de educación primaria de esas universidades organizan el estudio del área de español en tres direcciones: literatura infantil, didáctica de la lectoescritura y didáctica de la lengua española; tratadas desde diferentes perspectivas y niveles de profundización (Murillo, 2007). 
El abordaje metodológico de la literatura infantil muestra dos tradiciones teóricometodológicas diferentes: una dirigida hacia la educación literaria (UNA y UCR) y la otra como complemento del currículum escolar (UNED).

En el área de didáctica de la lectoescritura se estudian los fundamentos teóricos de la lectoescritura inicial, partiendo del estudio de los métodos tradicionales hasta llegar a los más recientes, con el afán de ilustrar al futuro educador acerca de la evolución del aprendizaje de la lectura y la escritura a través del tiempo.

En ese sentido se analizan críticamente los métodos existentes, con sus pro y sus contra, con el propósito de que el estudiante defina acertadamente cuál es el más apropiado para iniciar a los niños en la lectura y escritura convencionales.

La tercera área, didáctica de la lengua española, presenta una mayor diversidad en su tratamiento y, en consecuencia, desde el punto de vista teórico-metodológico es la más débil.

En primer término, el adecuado desarrollo de esta área supone un perfil específico del formador universitario, en el sentido de que debe generar y poseer conocimientos sobre el área de lengua y su didáctica, el medio escolar y el desarrollo del niño de 7 a 14 años, en diálogo académico continuo con sus pares. Este profesional debe ser capaz de definir la secuencialidad, profundidad y pertinencia de los aspectos por trabajar en lengua y literatura españolas en la educación primaria.

La didáctica de la lengua española en la educación primaria debe abarcar las cuatro macrohabilidades del lenguaje con sus respectivas microhabilidades, en consonancia con la etapa de desarrollo de los escolares. Estas deben tratarse como componentes con características propias y ofrecer al futuro educador las estrategias para integrar esos conocimientos en el aula (Prado, 2004 y Murillo, 2005).

Ahora bien, si el objetivo macro de la enseñanza de la lengua en la educación primaria es 'enriquecer la competencia comunicativa de los escolares', la enseñanza debe sustentarse en el enfoque comunicativo, el cual toma como punto de partida el capital lingüístico de los 
educandos y a partir de este orienta los procesos de intervención pedagógica hacia una competencia meta (Lomas, 2004).

Los programas de formación de maestros deben asegurar al futuro educador una rigurosa formación en el área de lengua, en tanto que se la considera como área instrumental para la adquisición de nuevos conocimientos y para la socialización. A manera de ejemplo, diríamos que deben manejarse las metodologías específicas para enseñar los diferentes aspectos de la expresión escrita: puntuación, ortografía, marcadores textuales, vocabulario, tipos de discurso y producción de textos (Cassany, 2000).

En suma, podría afirmarse que no se visualiza en la propuesta estatal, como un todo, un tratamiento de la lengua como eje instrumental para la socialización y adquisición de nuevos conocimientos. No obstante, el planteamiento teórico-metodológico más acertado es el de la UCR, ya que se sustenta en un enfoque comunicativo.

En consecuencia, los planes de formación inicial docente (universidades públicas y privadas) deberían considerar en el planteamiento del área de lengua, los siguientes aspectos:

1. Dotar a los estudiantes de la carrera de educación primaria de los conocimientos teóricos y prácticos necesarios para adquirir las habilidades y destrezas que demanda el proceso de construcción y comprensión de textos orales y escritos. El desarrollo adecuado de la competencia lingüística -oral y escrita- estimula en los futuros docentes el trabajo creativo con la producción y decodificación textuales, y les permite generar experiencias propias en el campo, a fin de preparase para guiar mejor el proceso de enseñanza-aprendizaje en los primeros dos ciclos de la educación general básica.

2. Tener presente que la lengua forma parte del currículum como sistema de comunicación en el aula y en la escuela; como medio de aprendizaje (aprender los conocimientos de las diferentes áreas) y como objeto de aprendizaje.

3. Enfatizar en el estudio de la expresión oral, comprensión oral, lectura, escritura, como macrohabilidades instrumentales para la socialización y la construcción y adquisición de nuevos conocimientos. Es preciso definir en el aula universitaria las microhabilidades 
asociadas a cada una de las macrohabilidades mencionadas y las metodologías específicas para su estudio, en correlación con la etapa de desarrollo y nivel escolar de los educandos. Igualmente, deben considerarse los conceptos de secuencialidad, profundidad y pertinencia de los contenidos asociados a cada microhabilidad a lo largo de toda la escolaridad.

4. Asumir el enfoque comunicativo como sustento teórico para lograr el objetivo esencial de la educación lingüística y literaria en la enseñanza primaria, implica que "el desarrollo de la competencia comunicativa (expresiva y comprensiva) de los alumnos y de las alumnas, es decir, de su capacidad para comprender y producir enunciados adecuados a intenciones diversas de comunicación en contextos comunicativos heterogéneos" (Murillo, 2005), sea una realidad en las aulas costarricenses.

5. Con fundamentación en la triada lengua, cultura e identidad, incorporar como temática de estudio el conocimiento general de las características prototípicas del español de Costa Rica y la necesidad de utilizar la variedad prestigiosa en el discurso pedagógico.

6. Siguiendo el principio de funcionalidad, se recomienda trabajar la producción escrita a partir de las diferentes tipologías textuales -descriptiva, explicativa, argumentativa, instructiva, narrativa, expositiva-, a fin de que el escolar las aplique a las diferentes áreas curriculares. "Desde la enseñanza-aprendizaje de la Lengua se han de poder delimitar unos espacios para llegar a describir, definir, justificar, argumentar. Entendemos que la progresiva apropiación por parte del alumnado de esas habilidades puede mejorar tanto su competencia comunicativa como la capacidad de reflexión sobre la lengua como instrumento (Palou y Prat, 2000, p. 171).

7. Estudiar desde una perspectiva crítica los enfoques de lectoescritura inicial y enfatizar en los abordajes actuales. "La concepción actual de lectura se realiza en el interior del marco teórico general que explica cómo los seres humanos interpretan la realidad, cómo procesan la información". (Colomer y Camps, 2000, p. 34) En este sentido se habla de tres modelos sobre el proceso de lectura que han surgido a lo largo de las últimas décadas: proceso ascendente, descendente e interactivo.

Las dos formas de proceder, de abajo arriba y de arriba abajo, quedan englobadas en la idea básica de que, cuando una persona lee, parte de la hipótesis de que el texto posee un significado y lo que busca a través tanto del descubrimiento de indicios 
visuales como de la activación de una serie de mecanismos mentales que le permiten atribuirle un sentido, es decir, entenderlo. Lo que el lector ve en el texto y lo que él mismo aporta son dos subprocesos simultáneos y en estrecha interdependencia. Esta visión del proceso constituye lo que se llama modelos interactivos de lectura. (Colomer y Camps, 2000, p. 35)

8. Abordar la literatura infantil desde el enfoque de la educación literaria. Tal y como lo enuncia Colomer (1995), la educación literaria tendrá como meta formar lectores competentes y con una aceptable competencia literaria, la cual trabaja en los niveles de comprensión y expresión: desarrollo de formas de expresión escrita y lectura comprensiva de textos literarios.

\subsection{Programación del aprendizaje lingüístico escolar, en atención a las competencias por desarrollar en cada nivel educativo}

En primera instancia es necesario plantear los objetivos que sustentarán el enfoque curricular del área de lengua en la escuela primaria costarricense.

Sobre la base de Lomas (2001), Prado Aragonés (2004), MEP (1995, 2001, 2003, 2005), Murillo $(2004,2005)$, se proponen los siguientes objetivos generales para la educación lingüística y literaria en la enseñanza primaria costarricense:

1. Comunicar oralmente ideas, experiencias y sentimientos, adoptando una actitud respetuosa ante las aportaciones de los otros y atendiendo las reglas propias del intercambio comunicativo.

2. Desarrollar las estrategias de comprensión oral necesarias para interpretar y analizar crítica y objetivamente los mensajes escuchados como producto de diferentes interacciones orales.

3. Expresar por escrito de forma coherente ideas, experiencias y sentimientos, teniendo en cuenta las características de las diferentes situaciones de comunicación y los aspectos normativos de la lengua.

4. Utilizar la lectura como fuente de placer, de información y de aprendizaje y como medio de perfeccionamiento y enriquecimiento lingüístico personal. 
5. Reflexionar sobre el uso de la lengua, mediante el establecimiento de relaciones entre los aspectos formales y los contextos e intenciones comunicativas a los que responden, para mejorar las propias producciones.

Los objetivos propuestos deben abordarse desde un enfoque comunicativo y funcional, considerando que el objetivo básico es el logro de la competencia comunicativa, entendida como el conjunto de conocimientos lingüísticos y de habilidades comunicativas que se van adquiriendo a lo largo del proceso de socialización de las personas.

Desde este enfoque y del marco teórico cognoscitivo constructivista se propondrán las orientaciones metodológicas y las estrategias de evaluación. Igualmente se definirán las competencias por desarrollar en los escolares, en cada ciclo, indicando los correspondientes niveles de profundidad.

Por otra parte, proponemos que la definición de las competencias meta, objeto de estudio en la educación básica -expresión oral, comprensión oral, lectura y escritura-, se describan por separado, con el propósito de que en la planificación micro no se invisibilice ninguna, como sucede regularmente en las aulas costarricenses. Además, esta metodología permitirá al docente y al planificador curricular visualizar la secuencialidad y profundidad de los diferentes componentes de lengua en cada período escolar y facilitará enormemente la valoración de los procesos que han de vivir el niño y la niña en el arduo transitar por el fascinante mundo de las letras y los significados hacia la meta: convertirse en usuarios competentes en su lengua materna.

\subsection{Práctica profesional docente que privilegie el enriquecimiento de la competencia comunicativa de los escolares, mediante la promoción de aprendizajes relevantes}

Las prácticas pedagógicas que se desarrollen en la escuela primaria deben sustentarse en un enfoque comunicativo y funcional que privilegie el aprendizaje constructivo, significativo y relevante. Todo ello con el objetivo de desarrollar habilidades y competencias que posibiliten la capacidad de interacción comunicativa de los escolares.

En el contexto de esta propuesta se entiende por competencia comunicativa: 
La competencia comunicativa es ese conjunto de conocimientos lingüísticos y de habilidades comunicativas que se van adquiriendo a lo largo del proceso de socialización de las personas (dentro y fuera de la escuela). A medida que nos vamos relacionando con otras personas, en contextos diversos, vamos adquiriendo y dominando los conocimientos lingüísticos, las destrezas comunicativas y las normas socioculturales que caracterizan los intercambios comunicativos en las diferentes situaciones de comunicación de la vida cotidiana.

Por ello al aprender a hablar una lengua no sólo aprendemos a utilizar la gramática de esa lengua sino también el modo más adecuado de usarla según las características de la situación de comunicación y de los interlocutores, según los fines que cada uno persiga en el intercambio comunicativo, según el tono (formal o informal) de la interacción, según el canal utilizado (oral, escrito, formas no verbales con los gestos ...), según el género discursivo (conversación espontánea, entrevista, exposición, narración, descripción, argumentación ...) y según las normas que rigen el tipo de situación comunicativa de la que se trate. Dicho de otra manera: al aprender a usar una lengua no sólo aprendemos a construir frases gramaticalmente correctas (Como subraya Chomsky) sino también a saber qué decir, a quién, cuándo y cómo decirlo y qué y cuándo callar. (Lomas, 2001, p. 142)

En el enfoque comunicativo la intervención didáctica se centra en actos de interacción pedagógica y el contenido es el uso de la misma lengua, la cual es medio de transmisión y objetivo final de aprendizaje.

Valga recordar que la didáctica de la lengua, como disciplina científica, ha basado su especificidad en la adquisición y desarrollo de la competencia comunicativa y en la "actualidad, se decanta hacia los enfoques centrados en el dominio de los usos comunicativos, a partir de la selección de contenidos de tipo nocional y funcional, de la diferenciación entre la dualidad de norma/uso y de la distinción entre empleo/uso" (p. 60).

\section{En consecuencia, debe considerarse}

el carácter progresivo del aprendizaje lingüístico; la adquisición y el aprendizaje lingüístico tienen un desarrollo progresivo, a través de estadios de dominio lingüístico (...); paralelamente esto indica que en la actividad formativa del lenguaje, en realidad, siempre se evalúan estadios, grados transitorios, de aprendizaje, momentos 
intermedios de un largo proceso de adquisición y perfeccionamiento lingüístico. (Cantero y Mendoza, 2003, p. 47)

Así las cosas, la valoración de la competencia lingüística solamente puede ser apreciada a partir de la puesta en práctica, o sea, del uso de la lengua en situaciones concretas de comunicación -oral o escrita-.

Por otra parte, la enseñanza tradicional de la lengua española se centró en la descripción de las estructuras lingüísticas, asumiendo diferentes niveles de profundidad, dependiendo del tramo escolar de que se tratara. Ese tipo de enseñanza enfatizó en el estudio de las normas gramaticales organizadas a través de diferentes esquemas analíticos; forma válida, desde el punto de vista teórico, pero no adecuada como procedimiento lógico y eficaz para enseñar y aprender a usar una lengua. Además, se insistió en aspectos preceptivos y, en consecuencia, se juzgó como vicio de lenguaje lo regional -considerando todos los niveles de la lengua- en la medida que se contrastaba con una variedad de prestigio, en nuestro caso como lengua de extensión con el español peninsular y, particularmente con las normas emitidas desde la Real Academia Española.

Por otra parte, esta manera de actuar traslada al aula escolar parte de las tareas propias del lingüista como profesional, o sea, estudiar la estructura interna de una lengua y proceder a su respetiva descripción. Este es, según nuestro entender, el sinsentido de la enseñanza gramatical de la lengua materna en la educación primaria.

Ahora bien, sustituir el planteamiento gramatical por la perspectiva interactiva-comunicativa implica:

- Pasar del metalenguaje a la producción y comprensión textuales.

- Priorizar el desarrollo de las competencias respecto de la enseñanza-aprendizaje de contenidos.

- Reemplazar al alumno receptor pasivo por el alumno constructor de conocimientos.

- Sustituir al magister ex cátedra por un guía en el proceso de construcción de conocimientos.

- Presentar materiales didácticos enfocados hacia la producción y construcción de conocimientos, en correspondencia con las competencias cultivadas en los estudiantes. 
- Desplazar los criterios de evaluación tradicionales por otros que respondan al desarrollo de las competencias comunicativas de los escolares, según su nivel evolutivo y en correspondencia con un tratamiento integrado y secuencial de las competencias específicas por desarrollar.

Esta posición teórica requiere de un educador conocedor de la variedad de lengua de sus alumnos y de los usos lingüísticos que debe promover, consciente de que el papel de la escuela como instancia formal en la que se estudian diferentes tipos de textos debe favorecer un ambiente de interacción e intercambio lingüísticos, donde los alumnos se expresen y desarrollen destrezas de comprensión oral y escrita. La mediación pedagógica, en consecuencia, debe estar guiada por personas que gusten de la lectura, de la escritura, estimulen el pensamiento crítico, el análisis y la reflexión sobre la lengua usada en la comunidad escolar y nacional.

Habrá también un cambio de actitud, de saberlo todo a saber solo un poco y a plantearse preguntas y buscar soluciones junto con los niños; cambio de perspectiva profesional, de la preocupación por un resultado a la ocupación por el proceso de desarrollo de las habilidades lingüísticas de los niños; de un conocedor del código lingüístico y su enseñanza de manera desvinculada de la realidad, a un maestro que parte de un abordaje funcional de la lengua y la incorpore como área instrumental para la adquisición de nuevos conocimientos; de una persona generalista a una especialista en el método comunicativo y en las didácticas específicas de la lectura, la expresión escrita, la expresión oral y la comprensión oral.

\subsection{Recursos didácticos graduados, pertinentes y accesibles}

Una de las competencias

para aprender a aprender es la capacidad para buscar, jerarquizar y organizar la información omnipresente que hallamos principalmente -aunque no exclusivamenteen Internet. (...) Hacer que el alumno aprenda a aprender es ponerlo delante de un ordenador, no para hacer de él un mero usuario, sino para enseñarle a que se sirva de ese instrumento y lo adapte a sus usos y su cultura. (UNESCO, 2005, p. 80) 
En consecuencia, uno de los objetivos será posibilitar los espacios interactivos necesarios, para que el estudiante desarrolle habilidades que le permitan encontrar la información buscada, haciendo uso de los recursos adecuados -físicos o electrónicos-

Los recursos didácticos estarán constituidos por bibliotecas (físicas o virtuales), instituciones públicas y privadas, zoológicos, parques, en fin, todo tipo de espacio que ofrezca información variada y permita el cumplimiento de los objetivos didácticos previamente definidos.

Por otra parte, dentro del aula es necesario contar con diccionarios graduados (que respondan a diferentes niveles educativos y elaborados sobre la base del capital lingüístico de los educandos costarricenses y el léxico meta de cada tramo de la educación obligatoria), enciclopedias, libros de literatura universal, periódicos, revistas, libros de texto que respondan a las competencias por desarrollar en la educación básica, una computadora con acceso a Internet, guías didácticas elaboradas por el maestro, entre otros.

En suma, tanto dentro como fuera del aula, el estudiante debe tener la posibilidad de interactuar con recursos diversos que le permitan ir construyendo los conocimientos de base y adquiriendo una cultura general, que le faciliten su transitar por la sociedad del conocimiento.

\subsection{Políticas claras de supervisión y acompañamiento del ejercicio profesional docente}

El documento Diagnóstico sobre la Formación de Docentes en Instituciones de Educación Superior en Costa Rica, (Sol, 2004, p. 56), concluye señalando que:

(...) a partir de 1970, la institucionalidad del sistema educativo costarricense cambia paulatinamente, hacia una desconcentración desordenada, y a una dispersión institucional.

Lo que predomina en esta nueva institucionalidad es la falta de coordinación institucional, la falta de políticas nacionales y la carencia de una rectoría del sistema.

La formación de maestros, ha sufrido de ese caótico desarrollo. Si bien, gana profesionalismo con la incorporación de la formación de docentes a las instituciones de educación superior o universidades, se diluye la mística y la cultura y el ambiente 
de formación integral de servicio formativo que existía en las normales. Esto se agrava con el vertiginoso desarrollo de las universidades privadas.

La dispersión de la oferta educativa tiene niveles alarmantes que no parecen corregirse con la introducción de propuestas o sistemas de evaluación y acreditación $(\ldots)$

La cita anterior evidencia la terrible situación que en materia de formación docente vive Costa Rica: sesenta y ocho universidades privadas y tres públicas ofreciendo planes de formación diversos.

La trayectoria académica de las universidades estatales y los procesos de autoevaluación interna de las carreras de formación inicial docente, tendientes al mejoramiento de la calidad profesional de los futuros docentes, aseguran a la sociedad costarricense la formación de educadores con sólidas bases académicas y humanas, fortalecidas por la proyección, en los planes de estudio, de los procesos de investigación y de acción social que realizan los formadores de formadores de esas instituciones.

Como contraparte, la dificultad de valorar cualitativamente la oferta en formación docente, ofrecida por las universidades privadas, debido a la carencia de políticas nacionales que definan las competencias necesarias para el ejercicio de la profesión docente, casi imposibilita la regulación, en materia de formación, en la dispar y amplia oferta para formación docente, que ha venido en aumento desde la década de los setenta.

En vista de lo anterior, deben establecerse políticas claras de supervisión y acompañamiento del ejercicio profesional en el área de la docencia, con el propósito de subsanar las carencias en la formación académica de muchos docentes en servicio.

Asumiendo las ideas planteadas por Marcelo (2004), consideramos que el país necesita, con urgencia, un programa para el desarrollo profesional en servicio que contemple dos líneas: la inserción profesional y el acompañamiento al desarrollo profesional de los docentes insertados en el sistema.

Este tipo de propuesta requiere de administradores de la educación con un profundo compromiso con la educación costarricense, pues ellos serán quienes, mediante la 
observación cotidiana del quehacer docente en las aulas, faciliten espacios para promover los procesos de reflexión académica en el interior de las instituciones educativas y coordinen iniciativas de formación específica en las áreas que lo requieran.

En consecuencia, el administrador de la educación debe poseer una fuerte formación académica, ética profesional y compromiso social; deben estar involucrados en procesos de formación permanente, con el propósito de que desarrollen destrezas de observación que les permitan hacer una lectura adecuada de los procesos de enseñanza y aprendizaje que se realizan en las instituciones educativas.

El educador, por su parte, debe ser un líder que promueva aprendizajes complejos:

Hemos de pensar que el liderazgo es inherente al papel del profesor como profesional, que los profesores tienen una responsabilidad que cumplir. (...) $Y$ es inherente al liderazgo la capacidad de innovación. Nos parece que una de las exigencias que la sociedad está haciendo a los profesores de enseñanza primaria y secundaria es la capacidad de seguir aprendiendo e innovando. (Marcelo, 2004, p. 554)

La era de la información nos provee de una cantidad de posibilidades que pueden considerarse al desarrollar programas de formación profesional en servicio y su respectivo acompañamiento. Las redes son una alternativa, pues constituyen una posibilidad de superar las limitaciones institucionales, animan a trabajar en equipo y posibilitan el crecimiento a partir de las experiencias vividas.

Las universidades estatales, en diferente medida, están comprometidas con los procesos de formación continua del educador y ofertan cursos, conferencias, talleres; desarrollan investigaciones que tienen como meta alimentar los procesos de formación en servicio e inclusive existen iniciativas específicas, por ejemplo, recientemente se conformó, en el seno del INIL-UCR, la comisión denominada "Pro mejoramiento de la lengua española en la educación costarricense", bajo la coordinación del Dr. Víctor MI. Sánchez Corrales, con el objetivo de ofrecer propuestas pertinentes al Ministerio de Educación Pública y apoyar en la medida que se le permita los procesos de planificación de la enseñanza de la lengua española en la educación primaria y secundaria costarricense. 


\section{Tareas pendientes}

Ofrecer una educación de calidad es la meta de todo sistema educativo, el nuestro no tiene por qué ser la excepción; por tanto, y con afán de construir juntos proyectos académicos y sociales que conduzcan al país hacia esa educación deseada, se proponen algunas tareas que competerían al Ministerio de Educación y otras a las universidades públicas.

\section{Ministerio de Educación Pública}

1. Conceptualizar y operacionalizar la enseñanza-aprendizaje de la lengua como área instrumental para la adquisición de conocimientos y la socialización, de tal forma que atraviese todo el currículum escolar.

2. Definir las competencias lingüísticas y literarias que se deben desarrollar en los niños en cada ciclo y nivel educativo, sobre la base de investigaciones que sustenten la correspondiente planificación.

3. Organizar un grupo de trabajo que se aboque a la reestructuración cualitativa de la enseñanza del español en la educación primaria y que redacte nuevos programas para I y II ciclos, en el marco de una política educativa que trascienda el gobierno de turno.

4. Crear mecanismos de supervisión y acompañamiento docente en el ejercicio profesional.

5. Suplir a las escuelas con material didáctico básico: diccionarios graduados, enciclopedias, libros de literatura universal, libros de texto y de actividades. Además, velar porque las bibliotecas escolares se constituyan en verdaderos espacios de promoción y animación de la lectura.

6. Convalidación inicial y revalidación de la calidad profesional de los educadores.

\section{Universidades estatales}

1. Formación de docentes con conocimientos sólidos en el área de lengua y su respectiva didáctica. 
2. Continuar con producción investigativa acerca del capital lingüístico de los escolares costarricenses y la práctica docente en esta área, para aportar insumos a la planificación lingüística curricular.

3. Constituirse en entes consultivos en materia de programación lingüística curricular y en la elaboración de materiales didácticos.

4. Ofrecer sistemáticamente espacios de formación continua a los educadores en servicio, aprovechando la infraestructura distribuida en todo el territorio nacional y las tecnologías de la información.

\section{Referencias}

Cassany, Daniel, Luna, Marta y Sanz, Gloria. (1997). Enseñar lengua. Barcelona: Graó.

Cantero Serena, Francisco José y Mendoza Fillola, Antonio. (2003) Conceptos básicos en Didáctica de la Lengua y la Literatura. En: Didáctica de la lengua y la literatura. Editorial Prentice Hall, España)

Colomer, Teresa y Camps, Anna. (1996/2000). Enseñar a leer, enseñar a comprender. Madrid: Celeste/M.E.C.

Lomas, Carlos. (2001). Cómo enseñar a hacer cosas con palabras (Vol. I y II). Barcelona: Paidós.

Lomas, Carlos. (2001). O valor das palabras (I) Falar, ler e escrever nas aulas. Ediciones ASA, Lisboa.

Marcelo, Carlos. (2004). Aprender a enseñar para la sociedad del conocimiento. Recuperado el 15 de junio de 2007, de http://www.ucm.es/BUCM/revistas/edu/11302496/articulos/RCED0101220531A.PDF

Ministerio de Educación Pública - Costa Rica. (2005). Programa de estudios. Español I y II ciclo de la Educación General Básica. San José: Departamento de publicaciones, Ministerio de Educación Pública.

Ministerio de Educación Pública - Costa Rica. (1995). Programa de estudios. Español I y II ciclo de la Educación General Básica. San José: Departamento de publicaciones, Ministerio de Educación Pública.

Ministerio de Educación Pública - Costa Rica. (1994). Política Educativa Hacia el Siglo XXI. Recuperado el 15 de marzo de 2007, de www.mep.go.cr. 
Ministerio de Educación Pública - Costa Rica. (1991). Programa de estudios. Español I y II ciclo de la Educación General Básica. San José: Departamento de publicaciones, Ministerio de Educación Pública.

Murillo, Marielos y Morales, Mauren. (2007). La investigación sobre el aprendizaje y la enseñanza de la lengua española en la educación primaria costarricense. Aportes de las universidades estatales y algunas tendencias latinoamericanas. Revista Electrónica Actualidades Investigativas en Educación. En prensa.

Murillo, Marielos (2007). La enseñanza de la lengua española en la educación primaria costarricense. Período 1995-2007. Análisis crítico. Káñina, Revista de Artes y Letras de la Universidad de Costa Rica. Entregado el 10 de agosto de 2007

Murillo, Marielos. (2005). La lectura en la escuela costarricense. Algunas reflexiones. Revista Electrónica Actualidades Investigativas en Educación, 5 (2). Disponible en http://revista.inie.ucr.ac.cr

Murillo, Marielos. (2005). Comunicarse y crecer: Análisis crítico del trabajo con la lectura, la escritura, la expresión y comprensión orales en la escuela costarricense. Káñina, Revista de Artes y Letras de la Universidad de Costa Rica, XXIX (1), 175-189.

Murillo, Marielos. (2004). Estudio sobre el lenguaje de los escolares costarricenses. EI léxico básico. La ortografía y sus características. Tesis doctoral. Universidad de Extremadura, España.

Prado Aragonés, Josefina. 2004. Didáctica de la lengua y la literatura para educar en el siglo XXI. Madrid: La Muralla.

Sol Arriaza, Ricardo. (2004). Diagnóstico sobre la Formación de Docentes en Instituciones de Educación Superior en Costa Rica. San José: IESAL/UNESCO.

UNESCO. (2005) Informe de Seguimiento de Educación para Todos en el Mundo. EI imperativo de la Calidad, 2005. Recuperado el 15 de mayo de 2007, de http://unesdoc.unesco.org/images/0015/001501/1501695.pdf 